\title{
Some exact and explicit solutions to a two- component, discrete, nonlinear Schrödinger model
}

\author{
İsmail Aslan
}

\begin{abstract}
Natural processes and phenomena often display discrete structure. The discrete nonlinear Schrödinger equations are used in both physics and biology to model periodic optical structures and energy transfer in proteins. In this study, we present a new application of the $\left(G^{\prime} / G\right)$-expansion method to special, coupled, discrete, nonlinear Schrödinger-type equations. This application is shown to be an effective tool for constructing solitary and periodic wave profiles with arbitrary parameters. In addition, we provide rational solutions that have not been explicitly computed before.
\end{abstract}

PACS Nos: 02.30.Jr, 05.45.Yv, 02.30.Ik

Résumé : Les processus et phénomènes physiques montrent souvent une structure discrète. Les équations discrètes non linéaires de Schrödinger sont utilisées en physique et en biologie pour modéliser des structures optiques périodiques et des transferts d'énergie dans les protéines. Ici, nous présentons une nouvelle application de la méthode de l'expansion $\left(G^{\prime} / G\right)$ aux équations spéciales du type de Schrödinger non linéaires discrètes et couplées. Nous montrons que cette application est un outil utile pour construire des profils ondulatoires, solitaires et périodiques, avec des paramètres arbitraires. De plus, nous en donnons des solutions rationnelles qui n'ont pas été obtenues précédemment de façon explicite.

[Traduit par la Rédaction]

\section{Introduction}

Nonlinear differential-difference equations (NDDEs) play a crucial role in different branches of applied nonlinear sciences, such as condensed matter physics, biophysics, and mechanical engineering, and in various physical problems, such as currents in electrical networks, atomic chains, and molecular crystals, and have become a focus of common concern. Because of their rich structures, NDDEs are also encountered in numerical simulations of soliton dynamics in high energy physics. Unlike difference equations, which are fully discretized, NDDEs are semidiscretized; some (or all) of their space variables are discretized, while time is usually kept continuous. Thus, they can be considered hybrid systems. Directly seeking exact solutions of NDDEs has attracted much attention in the study of their corresponding nonlinear phenomena because these solutions might give better insight into the physical aspects of the problems considered. Hence, many powerful and effective methods have been developed for tackling NDDEs. To make mention of a few; Hirota's bilinear method [1], the Exp-function method [2], the homotopy perturbation method [3], the Adomian decomposition method (ADM) - Padé technique [4], and the discrete Jacobi-sub equation method [5].

The discrete nonlinear Schrödinger (DNLS) equations and related nonlinear lattice models have been prominent in the description of numerous experiments in applied nonlinear sciences. The DNLS equations have also been implemented in a variety of other settings to make crucial predictions that have not yet been verified experimentally. The study of the DNLS equations dates back to theoretical work on biophysics in the early 1970s [6]. One of the most important generalizations of the DNLS equation is in the study of multicomponent versions of the model. Such models are relevant both from theoretical and experimental points of view. The coupling among the different components of the DNLS model can be linear or nonlinear (or both). As an extension of the DNLS model, we consider the case of the two-component DNLS model of the form

$$
\begin{array}{r}
i \frac{\mathrm{d} \phi_{n}(t)}{\mathrm{d} t}+\left[\phi_{n-1}(t)+\phi_{n+1}(t)\right]\left[1-\left(\left|\phi_{n}(t)\right|^{2}+\left|\psi_{n}(t)\right|^{2}\right)\right] \\
-2 \phi_{n}(t)=0 \\
i \frac{\mathrm{d} \psi_{n}(t)}{\mathrm{d} t}+\left[\psi_{n-1}(t)+\psi_{n+1}(t)\right]\left[1-\left(\left|\phi_{n}(t)\right|^{2}+\left|\psi_{n}(t)\right|^{2}\right)\right] \\
-2 \psi_{n}(t)=0
\end{array}
$$

where $\phi_{n}(t)=\phi(n, t)$ and $\psi_{n}(t)=\psi(n, t)$ are complex-valued functions for all integer values of the site index $n$. When $\phi_{n}(t) \equiv 0$ or $\psi_{n}(t) \equiv 0$, the system (1a), (1b) degenerates into an Ablowitz-Ladik model [7, 8], which is used in modelling diverse physical phenomena, including the dynamics of a harmonic lattice, self-trapping on a dimer, and pulse dynamics in nonlinear optics. See ref. 9 for more details on the physical relevance of this model.

In the literature, there exist some generic methods to construct exact and explict solutions to nonlinear evolution equations (NEEs). For example, the transformed rational function method [10], multiple Exp-function method [11], linear superposition principle [12], and symmetry algebra method

Received 16 May 2011. Accepted 3 August 2011. Published at www.nrcresearchpress.com/cjp on 23 August 2011.

İ. Aslan. Department of Mathematics, Izmir Institute of Technology, Urla, İzmir 35430, Turkey.

E-mail for correspondence: ismailaslan@iyte.edu.tr. 
[13]. In 2008, Wang et al. [14] proposed a powerful technique; the so-called $\left(G^{\prime} / G\right)$-expansion method, for solving nonlinear evolution equations. Based on Wang et al.'s pioneer work and their followers', the $\left(G^{\prime} / G\right)$-expansion method has attracted the attention of the research community and a number of studies refining the initial idea appear in the research literature [15-30]. The value of the $\left(G^{\prime} / G\right)$-expansion method lies in the fact that one treats NEEs by essentially linear methods. It is entirely algorithmic and transforms a nonlinear equation into an algebraic computation. If treated rigorously, the $\left(G^{\prime} / G\right)$-expansion method usually reveals three types of exact traveling wave solutions (in terms of hyperbolic, trigonometric, and rational functions) in a neat form. In fact, the transformed rational function method is simply one of the generalizations of the $\left(G^{\prime} / G\right)$-expansion method, but the multiple Exp-function method is much more general.

Our objective in this study is twofold: first, to introduce the $\left(G^{\prime} / G\right)$-expansion method for handling systems of NDDEs so that one can apply it to solving different types of problems arising in nonlinear science, and second, to illustrate its application to coupled, discrete, nonlinear Schrödinger equations for the first time. The rest of this paper is organized as follows: In Sect. 2, we present the key steps of the $\left(G^{\prime} / G\right)$-expansion method for NDDEs. In Sect. 3, we analyze our problem for discrete traveling wave solutions. In Sect. 4 , we give some concluding remarks.

\section{Methodology}

Let us consider a system of $M$ polynomial NDDEs in the form

$$
\begin{array}{r}
\Delta\left(\boldsymbol{u}_{\boldsymbol{n}+\boldsymbol{p}_{1}}(\boldsymbol{x}), \ldots, \boldsymbol{u}_{\boldsymbol{n}+\boldsymbol{p}_{k}}(\boldsymbol{x}), \ldots, \boldsymbol{u}_{\boldsymbol{n}+\boldsymbol{p}_{1}}^{\prime}(\boldsymbol{x}), \ldots, \boldsymbol{u}_{\boldsymbol{n}+\boldsymbol{p}_{k}}^{\prime}(\boldsymbol{x}),\right. \\
\left.\ldots, \boldsymbol{u}_{\boldsymbol{n}+\boldsymbol{p}_{1}}^{(r)}(\boldsymbol{x}), \ldots, \boldsymbol{u}_{\boldsymbol{n}+\boldsymbol{p}_{k}}^{(r)}(\boldsymbol{x})\right)=0
\end{array}
$$

in which the dependent variables, $\boldsymbol{u}_{\boldsymbol{n}}$, have $M$ components, $u_{i, \boldsymbol{n}}$, and so do its shifts; the continuous variable $\boldsymbol{x}$ has $N$ components, $x_{i}$; the discrete variable $\boldsymbol{n}$ has $Q$ components, $n_{j} ; \boldsymbol{p}_{s}$ $(s=1, \ldots, k)$ denotes shift vectors with $Q$ components; and $\boldsymbol{u}^{(r)}(\boldsymbol{x})$ is the collection of mixed derivative terms of order $r$.

Step 1. To search for traveling wave solutions of (2), we first make the wave transformation

$$
\begin{array}{r}
\boldsymbol{u}_{\boldsymbol{n}+\boldsymbol{p}_{s}}(\boldsymbol{x})=\boldsymbol{U}_{\boldsymbol{n}+\boldsymbol{p}_{s}}\left(\xi_{\boldsymbol{n}}\right) \quad \xi_{\boldsymbol{n}}=\sum_{i=1}^{Q} d_{i} n_{i}+\sum_{\substack{j=1 \\
(s=1,2, \ldots, k)}}^{N} c_{j} x_{j}+\zeta \\
(s=1,2)
\end{array}
$$

where the coefficients $c_{1}, c_{2}, \ldots, c_{N}, d_{1}, d_{2}, \ldots, d_{Q}$, and the phase $\zeta$ are all constants, and then convert (2) into

$$
\begin{array}{r}
\Delta\left(\boldsymbol{U}_{\boldsymbol{n}+\boldsymbol{p}_{1}}\left(\xi_{\boldsymbol{n}}\right), \ldots, \boldsymbol{U}_{\boldsymbol{n}+\boldsymbol{p}_{k}}\left(\xi_{\boldsymbol{n}}\right), \ldots, \boldsymbol{U}_{\boldsymbol{n}+\boldsymbol{p}_{1}}^{\prime}\left(\xi_{\boldsymbol{n}}\right), \ldots, \boldsymbol{U}_{\boldsymbol{n}+\boldsymbol{p}_{k}}^{\prime}\left(\xi_{\boldsymbol{n}}\right),\right. \\
\left.\ldots, \boldsymbol{U}_{\boldsymbol{n}+\boldsymbol{p}_{1}}^{(r)}\left(\xi_{\boldsymbol{n}}\right), \ldots, \boldsymbol{U}_{\boldsymbol{n}+\boldsymbol{p}_{k}}^{(r)}\left(\xi_{\boldsymbol{n}}\right)\right)=0
\end{array}
$$

Step 2. We initially guess the solution of (4) in the form

$$
\boldsymbol{U}_{\boldsymbol{n}}\left(\xi_{\boldsymbol{n}}\right)=\sum_{l=0}^{m} a_{l}\left(\frac{G^{\prime}\left(\xi_{\boldsymbol{n}}\right)}{G\left(\xi_{\boldsymbol{n}}\right)}\right)^{l} \quad a_{m} \neq 0
$$

where $m$ (a positive integer) and the $a_{i}$ s are constants to be determined, and $G\left(\xi_{n}\right)$ satisfies the linear equation
$G^{\prime \prime}\left(\xi_{n}\right)+\mu G\left(\xi_{n}\right)=0$

where $\mu$ is an arbitrary constant and the prime denotes the derivative with respect to $\xi_{n}$. The general solution of (6) is well known from the linear theory. So, we obtain the following cases:

$$
\frac{G^{\prime}\left(\xi_{n}\right)}{G\left(\xi_{n}\right)}=\sqrt{-\mu}\left[\frac{C_{1} \cosh \left(\sqrt{-\mu} \xi_{n}\right)+C_{2} \sinh \left(\sqrt{-\mu} \xi_{n}\right)}{C_{1} \sinh \left(\sqrt{-\mu} \xi_{n}\right)+C_{2} \cosh \left(\sqrt{-\mu} \xi_{n}\right)}\right]
$$

$$
\frac{G^{\prime}\left(\xi_{n}\right)}{G\left(\xi_{n}\right)}=\sqrt{\mu}\left[\frac{-C_{1} \sin \left(\sqrt{\mu} \xi_{n}\right)+C_{2} \cos \left(\sqrt{\mu} \xi_{n}\right)}{C_{1} \cos \left(\sqrt{\mu} \xi_{n}\right)+C_{2} \sin \left(\sqrt{\mu} \xi_{n}\right)}\right]_{\mu>0}
$$

$\frac{G^{\prime}\left(\xi_{n}\right)}{G\left(\xi_{n}\right)}=\frac{C_{1}}{C_{1} \xi_{n}+C_{2}} \quad \mu=0$

where $C_{1}$ and $C_{2}$ are arbitrary constants.

Step 3. By a straightforward calculation, we can get the identity

$\xi_{\boldsymbol{n}+\boldsymbol{p}_{s}}=\xi_{\boldsymbol{n}}+\varphi_{s} \quad \varphi_{s}=p_{s 1} d_{1}+p_{s 2} d_{2}+\ldots+p_{s Q} d_{Q}$

where $p_{s j}$ is the $j$ th component of the shift vector $\boldsymbol{p}_{s}$. Applying the trigonometric and hyperbolic function identities and considering (7a)-(7c) along with (8), we derive the shift functions as follows:

$$
\begin{gathered}
\boldsymbol{U}_{\boldsymbol{n}+\boldsymbol{p}_{s}}\left(\xi_{\boldsymbol{n}}\right)=\sum_{l=0}^{m} a_{l}\left[\frac{\sqrt{-\mu} G^{\prime}\left(\xi_{\boldsymbol{n}}\right)-\mu \tanh \left(\sqrt{-\mu} \varphi_{s}\right) G\left(\xi_{\boldsymbol{n}}\right)}{\sqrt{-\mu} G\left(\xi_{\boldsymbol{n}}\right)+\tanh \left(\sqrt{-\mu} \varphi_{s}\right) G^{\prime}\left(\xi_{\boldsymbol{n}}\right)}\right]^{l} \\
a_{m} \neq 0 \quad \mu<0 \quad \text { (9a) } \\
\boldsymbol{U}_{\boldsymbol{n}+\boldsymbol{p}_{s}}\left(\xi_{\boldsymbol{n}}\right)=\sum_{l=0}^{m} a_{l}\left[\frac{\sqrt{\mu} G^{\prime}\left(\xi_{\boldsymbol{n}}\right)-\mu \tan \left(\sqrt{\mu} \varphi_{s}\right) G\left(\xi_{\boldsymbol{n}}\right)}{\sqrt{\mu} G\left(\xi_{\boldsymbol{n}}\right)+\tan \left(\sqrt{\mu} \varphi_{s}\right) G^{\prime}\left(\xi_{\boldsymbol{n}}\right)}\right]^{l} \\
a_{m} \neq 0 \quad \mu>0 \quad(9 \mathrm{~b})
\end{gathered}
$$

$$
\boldsymbol{U}_{\boldsymbol{n}+\boldsymbol{p}_{s}}\left(\xi_{\boldsymbol{n}}\right)=\sum_{l=0}^{m} a_{l}\left(\frac{G^{\prime}\left(\xi_{\boldsymbol{n}}\right)}{G\left(\xi_{\boldsymbol{n}}\right)+\varphi_{s} G^{\prime}\left(\xi_{\boldsymbol{n}}\right)}\right)^{l} a_{m} \neq 0 \quad \mu=0
$$

Step 4. By means of the ansätz (5), we define the degree of $\boldsymbol{U}_{\boldsymbol{n}}\left(\xi_{\boldsymbol{n}}\right)$ as $D\left[\boldsymbol{U}_{\boldsymbol{n}}\left(\xi_{\boldsymbol{n}}\right)\right]=m$, which gives rise to the degree of other expressions as

$$
\begin{aligned}
& D\left[\boldsymbol{U}_{\boldsymbol{n}}^{(r)}\left(\xi_{\boldsymbol{n}}\right)\right]=m+r \\
& D\left[\left(\boldsymbol{U}_{\boldsymbol{n}}^{(r)}\left(\xi_{\boldsymbol{n}}\right)\right)^{\beta}\right]=\beta(m+r)
\end{aligned}
$$

$D\left[\left(\boldsymbol{U}_{\boldsymbol{n}}\left(\xi_{\boldsymbol{n}}\right)\right)^{\alpha}\left(\boldsymbol{U}_{\boldsymbol{n}}^{(r)}\left(\xi_{\boldsymbol{n}}\right)\right)^{\beta}\right]=\alpha m+\beta(m+r)$

Balancing the highest order nonlinear term(s) and the highest-order derivative term in $\boldsymbol{U}_{\boldsymbol{n}}\left(\xi_{\boldsymbol{n}}\right)$, we can easily deter- 
mine the degree, $m$, of (5) and (9a)-(9c) from (4). As $\boldsymbol{U}_{\boldsymbol{n}+\boldsymbol{p}_{s}}$ can be interpreted as being of degree zero in $\left[G^{\prime}\left(\xi_{n}\right) / G\left(\xi_{n}\right)\right]$, the leading terms of $\boldsymbol{U}_{\boldsymbol{n}+\boldsymbol{p}_{s}}\left(p_{s} \neq 0\right)$ will not affect the balancing procedure.

Step 5. Substituting the ansätze (5) and (9a)-(9c) along with (6) into (4), equating the coefficients of $\left[G^{\prime}\left(\xi_{n}\right) / G\left(\xi_{n}\right)\right]^{l}(l=0$, $1,2, \ldots)$ to zero, we obtain a system of nonlinear algebraic equations from which the undetermined constants $a_{l}, d_{i}, c_{j}$, and $\mu$ can be explicitly found. Substituting these results into (5), we can derive various kinds of discrete exact solutions to (2).

Remark 1. As is well known, the standard procedure of the $\left(G^{\prime} / G\right)$-expansion method takes the auxilary equation $G^{\prime \prime}+$ $\lambda G^{\prime}+\mu G=0$ into account. However, without loss of generality, we can take $\lambda=0$ for the sake of minimizing the number of parameters (see ref. 30) and reduce the auxiliary equation to $G^{\prime \prime}+\mu G=0$.

Remark 2. Contrary to the numerical computation of traveling wave solutions of NDDEs, our algorithm computes closed-form analytical solutions, which are polynomial in $\left(G^{\prime} / G\right)$. Besides their physical relevance, the knowledge of closed-form solutions of NDDEs facilitates accuracy testing of numerical solvers. If closed-form solutions exist for specific values of parameters and (or) initial conditions, then for the same conditions the numerically computed solutions agree with the analytical solutions.

\section{Application}

We consider the physical system described by the coupled, discrete, nonlinear Schrödinger equations (1a) and (1b). In fact, we can transform (1a)-(1b) into coupled nonlinear Schrödinger equations [31], using the continuous analogue of the equation if we asssume that the envelope functions $\phi_{n}(t), \psi_{n}(t)$ are slowly varying and set

$\phi(n, t)=\varepsilon \Phi\left(-\varepsilon n,-\varepsilon^{2} t\right)=\varepsilon \Phi(Z, T)$

$\psi(n, t)=\varepsilon \Psi\left(-\varepsilon n,-\varepsilon^{2} t\right)=\varepsilon \Psi(Z, T)$

where $\Phi(Z, T)$ and $\Psi(Z, T)$ are slowly varying functions of their arguments, $T$ and $Z$. Then, we have

$$
\begin{aligned}
& \phi(n \pm 1, t)=\varepsilon \Phi(Z \mp \varepsilon, T) \\
& =\varepsilon\left[\Phi(Z, T) \mp \varepsilon \frac{\mathrm{d} \Phi(Z, T)}{\mathrm{d} Z}+\frac{\varepsilon^{2}}{2} \frac{\mathrm{d}^{2} \Phi(Z, T)}{\mathrm{d} Z}+\mathcal{C}\left(\varepsilon^{3}\right)\right] \quad(11 \mathrm{a}) \\
& \psi(n \pm 1, t)=\varepsilon \Psi(Z \mp \varepsilon, T) \\
& =\varepsilon\left[\Psi(Z, T) \mp \varepsilon \frac{\mathrm{d} \Psi(Z, T)}{\mathrm{d} Z}+\frac{\varepsilon^{2}}{2} \frac{\mathrm{d}^{2} \Psi(Z, T)}{\mathrm{d} Z}+\mathcal{O}\left(\varepsilon^{3}\right)\right]
\end{aligned}
$$

Substituting these expressions into (1a) and (1b) and neglecting the higher order terms of $\varepsilon$, we derive the following expressions

$$
\begin{aligned}
& i \frac{\mathrm{d} \Phi(Z, T)}{\mathrm{d} Z}-\frac{\mathrm{d}^{2} \Phi(Z, T)}{\mathrm{d} Z}+2 \Phi(Z, T)\left(|\Phi|^{2}+|\Psi|^{2}\right)=0 \\
& i \frac{\mathrm{d} \Psi(Z, T)}{\mathrm{d} Z}-\frac{\mathrm{d}^{2} \Psi(Z, T)}{\mathrm{d} Z}+2 \Psi(Z, T)\left(|\Phi|^{2}+|\Psi|^{2}\right)=0
\end{aligned}
$$

which are just well-known, coupled, nonlinear Schrödinger equations [31]. However, quite often, discrete models are more realistic than continuous ones, and help to better understand the physics of the problem. Now, to solve (1a) and (1b), we make the traveling wave transformation

$$
\begin{array}{r}
\phi_{n}=\mathrm{e}^{i \theta_{n}} u_{n}\left(\xi_{n}\right) \quad \psi_{n}=\mathrm{e}^{i \theta_{n}} v_{n}\left(\xi_{n}\right) \begin{array}{r}
\theta_{n}=d_{1} n+c_{1} t+\zeta_{1} \\
\xi_{n}=d_{2} n+c_{2} t+\zeta_{2}
\end{array}
\end{array}
$$

and

$\phi_{n \pm 1}=\mathrm{e}^{i \theta_{n}} \mathrm{e}^{ \pm i d_{1}} u_{n \pm 1}\left(\xi_{n}\right) \quad \psi_{n \pm 1}=\mathrm{e}^{i \theta_{n}} \mathrm{e}^{ \pm i d_{1}} v_{n \pm 1}\left(\xi_{n}\right)$

where $u_{n}=u_{n}\left(\xi_{n}\right)$ and $v_{n}=v_{n}\left(\xi_{n}\right)$ are both real-valued functions, $d_{1}$ and $c_{1}$ are the wave number of the carrier wave and the frequency, $c_{2}$ and $d_{2}$ are related to the group velocity and the pulse width, and $\zeta_{1}$ and $\zeta_{2}$ denote the initial phases. If $\zeta_{1}$ and $\zeta_{2}$ are both zero, then the center of the soliton coincides with a lattice site. In this case, the solution becomes symmetric. If $\zeta_{1}$ and $\zeta_{2}$ are not zero then the center is located between lattice sites. In this instance, the solution becomes asymmetric. In this sense, the parameters $\zeta_{1}$ and $\zeta_{2}$ produce a continuous family of solitons with variable shape.

Next, using the Euler formula $\mathrm{e}^{ \pm i d_{1}}=\cos d_{1} \pm i \sin d_{1}$, substituting (13) and (14) into (1a) and (1b), and separating the real and the imaginary parts, we obtain the system

$-c_{1} u_{n}+\cos \left(d_{1}\right)\left(u_{n+1}+u_{n-1}\right)\left[1-\left(u_{n}^{2}+v_{n}^{2}\right)\right]-2 u_{n}=0$

$-c_{1} v_{n}+\cos \left(d_{1}\right)\left(v_{n+1}+v_{n-1}\right)\left[1-\left(u_{n}^{2}+v_{n}^{2}\right)\right]-2 v_{n}=0$

$c_{2} u_{n}^{\prime}+\sin \left(d_{1}\right)\left(u_{n+1}-u_{n-1}\right)\left[1-\left(u_{n}^{2}+v_{n}^{2}\right)\right]=0$

$c_{2} v_{n}^{\prime}+\sin \left(d_{1}\right)\left(v_{n+1}-v_{n-1}\right)\left[1-\left(u_{n}^{2}+v_{n}^{2}\right)\right]=0$

where the prime denotes the derivative with respect to $\xi_{n}$. We expand the solution of (15a)-(15d) in the form of (5). Balancing the linear term of the highest order with the highest nonlinear term in (15a)-(15d) leads to $m=1$. Thus, for the traveling wave solutions of (15a)-(15d), we assume the ansätz

$u_{n}=a_{0}+a_{1}\left(\frac{G^{\prime}\left(\xi_{n}\right)}{G\left(\xi_{n}\right)}\right) \quad a_{1} \neq 0$

$v_{n}=b_{0}+b_{1}\left(\frac{G^{\prime}\left(\xi_{n}\right)}{G\left(\xi_{n}\right)}\right) \quad b_{1} \neq 0$

where $G\left(\xi_{n}\right)$ is a solution of (6), while $a_{0}, a_{1}, b_{0}$, and $b_{1}$ are arbitrary constants to be determined.

Case 1. $\mu<0$.

In this case, we first derive the expressions $u_{n \pm 1}$ and $v_{n \pm 1}$ in accordance with (9a) and substitute them, along with (16a) and (16b), into (15a)-(15d). Then, clearing the denominator and setting the coefficients of $\left(G^{\prime} / G\right)^{l}(0 \leq l \leq 8)$ to zero, we derive a system of nonlinear algebraic equations for $a_{0}, a_{1}, b_{0}, b_{1}, d_{1}, d_{2}, c_{1}, c_{2}$, and $\mu$. Solving the system, we get the following solution sets: 


$$
\begin{array}{r}
\left\{a_{0}=0, \quad b_{0}=0, \quad c_{1}=2\left[-1+\cos \left(d_{1}\right)-\cos \left(d_{1}\right) \tanh ^{2}\left(\sqrt{-\mu} d_{2}\right)\right], c_{2}=-2 \sqrt{-\mu} \sin \left(d_{1}\right) \tanh \left(\sqrt{-\mu} d_{2}\right) / \mu\right. \\
b_{1}=\mp \sqrt{\left.\mu a_{1}^{2}+\tanh ^{2}\left(\sqrt{-\mu} d_{2}\right) / \sqrt{-\mu}\right\}}
\end{array}
$$

and the corresponding discrete hyperbolic functions, solutions to (1a) and (1b) as

$$
\begin{array}{r}
\phi_{n, 1}(t)=\sqrt{-\mu} a_{1}\left[\begin{array}{r}
C_{1} \cosh \left(\sqrt{-\mu} \xi_{n}\right)+C_{2} \sinh \left(\sqrt{-\mu} \xi_{n}\right) \\
C_{1} \sinh \left(\sqrt{-\mu} \xi_{n}\right)+C_{2} \cosh \left(\sqrt{-\mu} \xi_{n}\right)
\end{array}\right] \\
\quad \times \exp \left(i\left\{d_{1} n+2\left[-1+\cos \left(d_{1}\right)-\cos \left(d_{1}\right) \tanh ^{2}\left(\sqrt{-\mu} d_{2}\right)\right] t+\zeta_{1}\right\}\right)
\end{array}
$$

where

$\xi_{n}=d_{2} n-\frac{2 \sqrt{-\mu} \sin \left(d_{1}\right) \tanh \left(\sqrt{-\mu} d_{2}\right)}{\mu} t+\zeta_{2}$

and $\mu(<0), a_{1}, d_{1}, d_{2}, \zeta_{1}, \zeta_{2}, C_{1}$, and $C_{2}$ remain arbitrary.

As a particular example, if we let $C_{1}=0$ and $C_{2} \neq 0$ or we let $C_{1} \neq 0$ and $C_{2}=0$ in (18a) and (18b), respectively, then we get formal, discrete, solitary wave solutions to (1a) and (1b) as follows:

$$
\begin{aligned}
& \phi_{n, 2}(t)=\sqrt{-\mu} a_{1} \tanh \left\{\sqrt{-\mu}\left[d_{2} n-\frac{2 \sqrt{-\mu} \sin \left(d_{1}\right) \tanh \left(\sqrt{-\mu} d_{2}\right)}{\mu} t+\zeta_{2}\right]\right\} \\
& \times \exp \left(i\left\{d_{1} n+2\left[-1+\cos \left(d_{1}\right)-\cos \left(d_{1}\right) \tanh ^{2}\left(\sqrt{-\mu} d_{2}\right)\right] t+\zeta_{1}\right\}\right) \\
& \begin{aligned}
& \psi_{n, 2}^{\mp}(t)=\mp \sqrt{\mu a_{1}^{2}+\tanh ^{2}\left(\sqrt{-\mu} d_{2}\right)} \tanh \left\{\sqrt{-\mu}\left[d_{2} n-\frac{2 \sqrt{-\mu} \sin \left(d_{1}\right) \tanh \left(\sqrt{-\mu} d_{2}\right)}{\mu} t+\zeta_{2}\right]\right\} \\
& \times \exp \left(i\left\{d_{1} n+2\left[-1+\cos \left(d_{1}\right)-\cos \left(d_{1}\right) \tanh ^{2}\left(\sqrt{-\mu} d_{2}\right)\right] t+\zeta_{1}\right\}\right)
\end{aligned} \\
& \phi_{n, 3}(t)=\sqrt{-\mu} a_{1} \operatorname{coth}\left(\sqrt{-\mu}\left[d_{2} n-\frac{2 \sqrt{-\mu} \sin \left(d_{1}\right) \tanh \left(\sqrt{-\mu} d_{2}\right)}{\mu} t+\zeta_{2}\right]\right) \\
& \times \exp \left(i\left\{d_{1} n+2\left[-1+\cos \left(d_{1}\right)-\cos \left(d_{1}\right) \tanh ^{2}\left(\sqrt{-\mu} d_{2}\right)\right] t+\zeta_{1}\right\}\right) \\
& \psi_{n, 3}^{\mp}(t)=\mp \sqrt{\mu a_{1}^{2}+\tanh ^{2}\left(\sqrt{-\mu} d_{2}\right)} \operatorname{coth}\left\{\sqrt{-\mu}\left[d_{2} n-\frac{2 \sqrt{-\mu} \sin \left(d_{1}\right) \tanh \left(\sqrt{-\mu} d_{2}\right)}{\mu} t+\zeta_{2}\right]\right\} \\
& \times \exp \left(i\left\{d_{1} n+2\left[-1+\cos \left(d_{1}\right)-\cos \left(d_{1}\right) \tanh ^{2}\left(\sqrt{-\mu} d_{2}\right)\right] t+\zeta_{1}\right\}\right)
\end{aligned}
$$

where $\mu(<0), a_{1}, d_{1}, d_{2}, \zeta_{1}$, and $\zeta_{2}$ are arbitrary constants.

Case 2. $\mu>0$.

In this case, we first derive the expressions $u_{n \pm 1}$ and $v_{n \pm 1}$ in accordance with (9b) and substitute them along with (16a) and (16b) into (15a)-(15d). Then, clearing the denominator and setting the coefficients of $\left(G^{\prime} / G\right)^{l}(0 \leq l \leq 8)$ to zero, we derive a system of 
nonlinear algebraic equations for $a_{0}, a_{1}, b_{0}, b_{1}, d_{1}, d_{2}, c_{1}, c_{2}$, and $\mu$. Solving the system, we get the following solution sets

$$
\begin{array}{r}
\left\{a_{0}=0, \quad b_{0}=0, c_{1}=2\left[-1+\cos \left(d_{1}\right)+\cos \left(d_{1}\right) \tan ^{2}\left(\sqrt{\mu} d_{2}\right)\right], c_{2}=2 \sin \left(d_{1}\right) \tan \left(\sqrt{\mu} d_{2}\right) / \sqrt{\mu},\right. \\
b_{1}=\mp \sqrt{\left.\tan ^{2}\left(\sqrt{\mu} d_{2}\right)-\mu a_{1}^{2} / \sqrt{\mu}\right\}}
\end{array}
$$

and the corresponding discrete trigonometric function solutions to (1a) and (1b) as

$$
\begin{aligned}
& \phi_{n, 4}(t)=\sqrt{\mu} a_{1}\left[\frac{-C_{1} \sin \left(\sqrt{\mu} \xi_{n}\right)+C_{2} \cos \left(\sqrt{\mu} \xi_{n}\right)}{C_{1} \cos \left(\sqrt{\mu} \xi_{n}\right)+C_{2} \sin \left(\sqrt{\mu} \xi_{n}\right)}\right] \\
& \times \exp \left(i\left\{d_{1} n+2\left[-1+\cos \left(d_{1}\right)+\cos \left(d_{1}\right) \tan ^{2}\left(\sqrt{\mu} d_{2}\right)\right] t+\zeta_{1}\right\}\right) \quad(22 \mathrm{a}) \\
& \begin{array}{r}
\psi_{n, 4}^{\mp}(t)=\mp \sqrt{\tan ^{2}\left(\sqrt{\mu} d_{2}\right)-\mu a_{1}^{2}}\left[\frac{-C_{1} \sin \left(\sqrt{\mu} \xi_{n}\right)+C_{2} \cos \left(\sqrt{\mu} \xi_{n}\right)}{C_{1} \cos \left(\sqrt{\mu} \xi_{n}\right)+C_{2} \sin \left(\sqrt{\mu} \xi_{n}\right)}\right] \\
\times \exp \left(i\left\{d_{1} n+2\left[-1+\cos \left(d_{1}\right)+\cos \left(d_{1}\right) \tan ^{2}\left(\sqrt{\mu} d_{2}\right)\right] t+\zeta_{1}\right\}\right)
\end{array}
\end{aligned}
$$

where

$\xi_{n}=d_{2} n+\frac{2 \sin \left(d_{1}\right) \tan \left(\sqrt{\mu} d_{2}\right)}{\sqrt{\mu}} t+\zeta_{2}$

and $\mu(<0), a_{1}, d_{1}, d_{2}, \zeta_{1}, \zeta_{2}, C_{1}$, and $C_{2}$ remain arbitrary.

As a particular example, if we take either $C_{1}=0$ and $C_{2} \neq 0$ or $C_{1} \neq 0$ and $C_{2}=0$ in expressions (22a) and (22b), respectively, then we get formal, discrete, periodic wave solutions to (1a) and (1b) as follows:

$$
\begin{aligned}
& \phi_{n, 5}(t)=\sqrt{\mu} a_{1} \cot \left\{\sqrt{\mu}\left[d_{2} n+\frac{2 \sin \left(d_{1}\right) \tan \left(\sqrt{\mu} d_{2}\right)}{\sqrt{\mu}} t+\zeta_{2}\right]\right\} \\
& \times \exp \left(i\left\{d_{1} n+2\left[-1+\cos \left(d_{1}\right)+\cos \left(d_{1}\right) \tan ^{2}\left(\sqrt{\mu} d_{2}\right)\right] t+\zeta_{1}\right\}\right) \\
& \psi_{n, 5}^{\mp}(t)=\mp \sqrt{\tan ^{2}\left(\sqrt{\mu} d_{2}\right)-\mu a_{1}^{2}} \cot \left\{\sqrt { \mu } \left[\begin{array}{r}
\left.\left.d_{2} n+\frac{2 \sin \left(d_{1}\right) \tan \left(\sqrt{\mu} d_{2}\right)}{\sqrt{\mu}} t+\zeta_{2}\right]\right\} \\
\times \exp \left(i\left\{d_{1} n+2\left[-1+\cos \left(d_{1}\right)+\cos \left(d_{1}\right) \tan ^{2}\left(\sqrt{\mu} d_{2}\right)\right] t+\zeta_{1}\right\}\right)
\end{array}\right.\right.
\end{aligned}
$$

$\phi_{n, 6}(t)=-\sqrt{\mu} a_{1} \tan \left\{\sqrt{\mu}\left[d_{2} n+\frac{2 \sin \left(d_{1}\right) \tan \left(\sqrt{\mu} d_{2}\right)}{\sqrt{\mu}} t+\zeta_{2}\right]\right\}$

$$
\times \exp \left(i\left\{d_{1} n+2\left[-1+\cos \left(d_{1}\right)+\cos \left(d_{1}\right) \tan ^{2}\left(\sqrt{\mu} d_{2}\right)\right] t+\zeta_{1}\right\}\right)
$$

$$
\begin{aligned}
& \psi_{n, 6}^{\mp}(t)= \pm \sqrt{\tan ^{2}\left(\sqrt{\mu} d_{2}\right)-\mu a_{1}^{2}} \tan \left\{\sqrt{\mu}\left[d_{2} n+\frac{2 \sin \left(d_{1}\right) \tan \left(\sqrt{\mu} d_{2}\right)}{\sqrt{\mu}} t+\zeta_{2}\right]\right\} \\
& \times \exp \left(i\left\{d_{1} n+2\left[-1+\cos \left(d_{1}\right)+\cos \left(d_{1}\right) \tan ^{2}\left(\sqrt{\mu} d_{2}\right)\right] t+\zeta_{1}\right\}\right)
\end{aligned}
$$


where $\mu(<0), a_{1}, d_{1}, d_{2}, \zeta_{1}$, and $\zeta_{2}$ are arbitrary constants.

Case 3. $\mu=0$.

In this case, we first derive the expressions $u_{n \pm 1}$ and $v_{n \pm 1}$ in accordance with (9c) and substitute them along with (16a) and (16b) into (15a) and (15b). Then, clearing the denominator and setting the coefficients of $\left(G^{\prime} / G\right)^{l}(0 \leq l \leq 7)$ to zero, we derive a system of nonlinear algebraic equations for $a_{0}, a_{1}, b_{0}, b_{1}$, $d_{1}, d_{2}, c_{1}$, and $c_{2}$. Solving the system, we get the following solution sets:

$$
\begin{aligned}
&\left\{c_{1}=2\left[-1+\cos \left(d_{1}\right)\right], \quad c_{2}=2 d_{2} \sin \left(d_{1}\right),\right. \\
&\left.a_{1}=\mp \sqrt{d_{2}^{2}-b_{1}^{2}}, \quad b_{0}=0, \quad a_{0}=0\right\}
\end{aligned}
$$

and the corresponding discrete rational function solutions to (1a) and (1b) as

$$
\begin{aligned}
\varphi_{n, 7}^{\mp}(t)=\mp & \sqrt{d_{2}^{2}-b_{1}^{2}}\left\{\frac{C_{1}}{C_{1}\left[d_{2} n+2 d_{2} \sin \left(d_{1}\right) t+\zeta_{2}\right]+C_{2}}\right\} \\
& \times \exp \left(i\left\{d_{1} n+2\left[-1+\cos \left(d_{1}\right)\right] t+\zeta_{1}\right\}\right) \\
\psi_{n, 7}(t)=b_{1} & \left\{\frac{C_{1}}{C_{1}\left[d_{2} n+2 d_{2} \sin \left(d_{1}\right) t+\zeta_{2}\right]+C_{2}}\right\} \\
& \times \exp \left(i\left\{d_{1} n+2\left[-1+\cos \left(d_{1}\right)\right] t+\zeta_{1}\right\}\right)
\end{aligned}
$$

where $b_{1}, d_{1}, d_{2}, \zeta_{1}, \zeta_{2}, C_{1}$, and $C_{2}$ remain arbitrary.

Remark 3. It is an important fact that one should be aware of the limitations of each of the existing methods. There is no guarantee that they will succeed for a specialized nonlinear problem. The $\left(G^{\prime} / G\right)$-expansion method assumes that the solution of the equation is in polynomial form with many parameters, and thus it sometimes leads to inconsistent nonlinear algebraic systems. However, we have encountered no such difficulty while working with (1a) and (1b).

\section{Conclusion}

We successfully utilized the $\left(G^{\prime} / G\right)$-expansion method for coupled, discrete, nonlinear, Schrödinger equations. Various kinds of exact solutions with more free parameters were constructed. New rational solutions were also obtained. The explicit construction of our solutions involved a large amount of tedious algebra and calculus. The use of a computer algebra system was necessary to carry out the lengthy (but straightforward) computations. The resulting traveling wave solutions are tested by substitution into the original equation; this provides an extra measure of confidence in the results. We foresee that the results of this study have the potential to be used as test problems for numerical comparison purposes. The method is straightforward, concise, and its potential for applications to other types of NDDEs is promising.

\section{References}

1. X.B. Hu and W.X. Ma. Phys. Lett. A, 293, 161 (2002). doi:10. 1016/S0375-9601(01)00850-7.
2. S.D. Zhu. Int. J. Nonlinear Sci. Numer. Simul. 8, 461 (2007). doi:10.1515/IJNSNS.2007.8.3.461.

3. S.D. Zhu, Y.M. Chu, and S.L. Qiu. Comput. Math. Appl. 58, 2398 (2009). doi:10.1016/j.camwa.2009.03.048.

4. P. Yang, Y. Chen, and Z.B. Li. Appl. Math. Comput. 210, 362 (2009). doi:10.1016/j.amc.2009.01.010.

5. H. Wang and H. Jia. Math. Methods Appl. Sci. 33, 1661 (2010). doi:10.1002/mma.1275.

6. A.S. Davydov. J. Theor. Biol. 38, 559 (1973). doi:10.1016/ 0022-5193(73)90256-7. PMID:4266326.

7. M.J. Ablowitz and J.F. Ladik. Stud. Appl. Math. 55, 213 (1976).

8. C.Q. Dai and J.F. Zhang. Int. J. Mod. Phys. B, 19, 2129 (2005). doi:10.1142/S0217979205029778.

9. P.G. Kevrekidis. The discrete nonlinear Schrödinger equation. Springer-Verlag, Berlin Heidelberg. 2009.

10. W.X. Ma and J.H. Lee. Chaos Solitons Fractals, 42, 1356 (2009). doi:10.1016/j.chaos.2009.03.043.

11. W.X. Ma, T. Huang, and Y. Zhang. Phys. Scr. 82, 065003 (2010). doi:10.1088/0031-8949/82/06/065003.

12. W.X. Ma and E. Fan. Comput. Math. Appl. 61, 950 (2011). doi:10.1016/j.camwa.2010.12.043.

13. W.X. Ma and M. Chen. Appl. Math. Comput. 215, 2835 (2009). doi:10.1016/j.amc.2009.09.024

14. M. Wang, X. Li, and J. Zhang. Phys. Lett. A, 372, 417 (2008). doi:10.1016/j.physleta.2007.07.051.

15. M. Wang, J. Zhang, and X. Li. Appl. Math. Comput. 206, 321 (2008). doi:10.1016/j.amc.2008.08.045.

16. L. Ling-Xiao and W. Ming-Liang. Appl. Math. Comput. 208, 440 (2009). doi:10.1016/j.amc.2008.12.005.

17. G. Ebadi and A. Biswas. J. Franklin Inst. 347, 1391 (2010). doi:10.1016/j.jfranklin.2010.05.013.

18. İ. Aslan and T. Öziş. Appl. Math. Comput. 209, 425 (2009). doi:10.1016/j.amc.2008.12.064.

19. İ. Aslan and T. Öziş. Appl. Math. Comput. 211, 531 (2009). doi:10.1016/j.amc.2009.01.075.

20. T. Öziş and İ. Aslan. Z. Naturforsch. A, 64a, 15 (2009).

21. T. Öziş and İ. Aslan. Commun. Theor. Phys. 51, 577 (2009). doi:10.1088/0253-6102/51/4/01.

22. İ. Aslan. Appl. Math. Comput. 215, 857 (2009). doi:10.1016/j. amc.2009.05.038.

23. T. Öziş and İ. Aslan. Appl. Math. Comput. 216, 2360 (2010). doi:10.1016/j.amc.2010.03.081.

24. İ. Aslan. Int. J. Nonlinear Sci. Numer. Simul. 15, 1967 (2010). doi:10.1016/j.cnsns.2009.08.008.

25. S. Zhang, W. Wang, and J. Tong. Appl. Math. Comput. 209, 399 (2009). doi:10.1016/j.amc.2008.12.068.

26. S. Zhang, L. Dong, J. Ba, and Y. Sun. Phys. Lett. A, 373, 905 (2009). doi:10.1016/j.physleta.2009.01.018.

27. İ. Aslan. Appl. Math. Comput. 215, 3140 (2009). doi:10.1016/ j.amc.2009.09.056.

28. İ. Aslan. Appl. Math. Comput. 216, 2778 (2010). doi:10.1016/ j.amc.2010.03.124.

29. İ. Aslan. J. Phys. A: Math. Theor. 43, 395207 (2010). doi:10. 1088/1751-8113/43/39/395207.

30. İ. Aslan. Appl. Math. Comput. 217, 937 (2010). doi:10.1016/j. amc.2010.05.097.

31. A.V. Buryak, Y.S. Kivshar, and D.F. Parker. Phys. Lett. A, 215, 57 (1996). doi:10.1016/0375-9601(96)00208-3. 